\title{
Lidil
}

Revue de linguistique et de didactique des langues

43 | 2011

Le rapport au savoir dans les discours professionnels

\section{Les savoirs convoqués par les formateurs dans les rapports de visite de stages de professeurs des écoles}

Place of knowledge and student teachers in the inspection reports written by the first degree teacher trainers

Fabienne Rondelli et Anne Leclaire-Halté

\section{OpenEdition}

Journals

Édition électronique

URL : http://journals.openedition.org/lidil/3112

DOI : $10.4000 /$ lidil.3112

ISSN : $1960-6052$

Éditeur

UGA Éditions/Université Grenoble Alpes

Édition imprimée

Date de publication : 30 mai 2011

Pagination : 89-102

ISBN : 978-2-84310-201-1

ISSN : $1146-6480$

\section{Référence électronique}

Fabienne Rondelli et Anne Leclaire-Halté, « Les savoirs convoqués par les formateurs dans les rapports de visite de stages de professeurs des écoles », Lidil [En ligne], 43 | 2011, mis en ligne le 30 novembre 2012, consulté le 01 mai 2019. URL : http://journals.openedition.org/lidil/3112 ; DOI : 10.4000/lidil.3112 


\title{
Les savoirs convoqués par les formateurs dans les rapports de visite de stages de professeurs des écoles
}

\author{
Fabienne Rondelli* et Anne Leclaire-Halté**
}

\section{RÉSUMÉ}

Cet article examine un genre de discours professionnel écrit caractérisé par de grandes variations rédactionnelles dans un cadre pourtant contraint, et par la pluralité de ses enjeux communicationnels : le rapport de visite tel qu'il est rédigé à l'IUFM par les membres d'une communauté socio-discursive, celle des formateurs d'enseignants du premier degré. Dans ce rapport de visite, les formateurs s'appuient sur des savoirs à/pour enseigner : lesquels ? Ce premier point est traité grâce à l'analyse d'un corpus de douze rapports de visite en cycle 3. Les formateurs convoquent aussi des savoirs sur l'acte de former, qui se lisent, comme le montre l'observation plus précise de deux exemples tirés du corpus, dans leurs choix énonciatifs, essentiellement par leur mode de désignation des acteurs et la tension entre discours expositif et discours argumentatif.

\section{ABSTRACT}

This article examines a genre of written professional discourse that is characterized by large editorial changes (yet constrained within) and by the plurality of its communicational issues: this discourse represents a visit report as written by the IUFM members of socio-discursive community, notably by the first degree teacher trainers. In this inspection report, trainers rely on the knowledge for teaching: so what is this knowledge? The first point is examined by means of corpus analysis, including about 10 visit reports of cycle 3. Teachers call also for knowledge about the act of training, which manifests itself (as shown by close observation of two examples extracted from the corpus) through enunciative choices, mainly by their mode of designation of actors and the tension between expositive and argumentative discourse.

* Université Paul Verlaine-Metz, Laboratoire CELTED.

** Université Henri Poincaré, IUFM de Lorraine, Laboratoire CELTED. 
Le dispositif de formation des professeurs des écoles stagiaires en IUFM (Institut universitaire de formation des Maitres), jusqu'à l'année universitaire 2009-2010, était fondé sur un principe directeur : l'articulation théorie-pratique. Cette orientation générale s'actualisait dans divers dispositifs, dont le stage en responsabilité, qui consistait en la prise en charge totale et singulière d'une classe. L'accompagnement par l'Institut se concrétisait, quant à lui, par des visites ponctuelles de formateurs qui donnaient lieu, chacune, à un rapport rédigé par ces mêmes formateurs. La rédaction de ce rapport relevait d'une pratique routinisée plus que d'une pratique émergente.

Certes, avec le processus de mastérisation, le rapport de visite est un objet sans doute institutionnellement moins central au moment de la préparation au concours en IUFM, mais d'une part, il perdure dans l'institution, du moins dans certaines académies, d'autre part, il reste un outil décisif de formation et de validation dans le cadre de l'année de stage du professeur des écoles lauréat au concours.

Dans ce cadre, nous avons choisi de soumettre cet écrit à notre examen parce qu'il semble particulièrement complexe et paradoxal. Sa complexité est double et tient d'une part au positionnement que le scripteur doit adopter en répondant à des objectifs de compte rendu descriptif, de conseil et d'évaluation sommative, et d'autre part, à la diversité des destinataires de l'écrit. La dimension paradoxale réside dans l'imposition d'un cadre d'écriture normé et néanmoins visiblement peu contraignant pour les scripteurs. Nous nous autorisons d'autant plus ce jugement que nous avons fait partie, ou faisons encore partie, des scripteurs en question.

Après avoir précisé le genre de discours professionnel qu'est le rapport de visite, nous poserons la question des savoirs sur lesquels s'appuient les formateurs pour rendre compte de la pratique des stagiaires.

\section{Inscription du genre rapport de visite dans un cadre institutionnel et communicationnel}

\section{Un genre de discours professionnel...}

Le rapport de visite s'inscrit dans le cadre très général des écrits professionnels. Cet écrit peut être qualifié de «genre de discours» dans le sens où il existe du fait d'une interaction discursive à la fois singulière et collective. Elle est singulière dans la mesure où un formateur et un formé se trouvent réunis dans une situation vécue unique : un profes- 
seur des écoles stagiaire fait la classe, est observé par un formateur qui, à chaud, mène un entretien sur la pratique vue et, à froid, rédige une fiche «mémoire» du temps d'observation-formation. Elle est collective dans le sens où le formateur, riche de la mémoire discursive de son groupe professionnel, rédige la fiche en fonction de caractéristiques pragmatiques, énonciatives, thématiques, structurelles qui lui sont disponibles mais aussi imposées par la pré-structuration du document.

Cette tension entre singularités et constantes caractérise les genres de discours :

Les genres de discours sont un moyen d'aborder la diversité socioculturellement réglée des pratiques discursives humaines. Le concept de discours rattache le singulier du texte à des catégories historiques, des «airs de famille». Sur une échelle qui va de l'identité et de la soumission au contraste et à la subversion, un texte renvoie à la chaine des discours qui circulent dans le champ culturel d'une formation sociodiscursive. (Adam, 2005, p. 28)

\section{... dans un cadre institutionnel donné mais soumis à variations...}

Dans l'académie où nous avons prélevé le corpus, les fiches supports du rapport de visite ont été élaborées par la commission chargée du premier degré de l'IUFM. Un seul modèle a cours dans l'ensemble des sites de l'académie. Il se compose de huit rubriques :

- conditions d'exercice;

- préparation et conception de situations;

- conduite de séances;

- relation pédagogique;

- analyse de la pratique;

- éléments de compétences (connaissances, capacités, attitudes) repérés comme étant déjà maitrisés ;

- éléments de compétences (connaissances, capacités, attitudes) repérés comme étant à travailler en priorité ;

- conseils, recommandations, consignes de travail pour la suite de la formation.

L'intitulé des rubriques semble inviter à :

- des focalisations plus ou moins resserrées sur un aspect de la pratique de classe («préparation et conception des situations »vs «relation pédagogique »); 
- un choix, selon les rubriques, entre description ou prise de position («conditions d'exercice» $v s$ «analyse de la pratique»).

On remarque aussi que, si le terme «évaluation» n'apparait nulle part, l'évaluation est cependant sollicitée dans les deux rubriques faisant référence aux éléments de compétences attendus en fin de formation. La dernière rubrique évoque, elle, explicitement une perspective formative et ne semble pas demander une récapitulation sous forme de bilan ${ }^{1}$.

Le document apparait donc très normé, structuré, invitant chaque formateur à une rédaction proche de celle de l'ensemble de ses collègues. En fait il n'en est rien. De grandes variations dans la façon de remplir une même rubrique sont repérables. Par exemple, la rubrique «Conduite de séances» que nous explorerons en détail dans la suite de notre propos varie, dans le corpus que nous avons réuni et que nous présentons plus loin, sur les points suivants :

- longueur (entre 8 lignes et 53 lignes);

- mise en page (mise en paragraphes ou non, jeux typographiques ou non);

- contenus : les disciplines, les objectifs, l'activité du maitre, des élèves sont présentés / développés ou non;

- actes de discours : le formateur peut choisir une modalité de discours à dominante expositive, il peut aussi à la fois exposer et s'inscrire dans une modalité verdictive.

Ce premier regard confirme qu'un document très cadré peut cependant être l'inducteur de rédactions assez peu semblables, alors que les locuteurs appartiennent à une communauté socio-discursive commune, celle des formateurs d'enseignants du premier degré.

\section{... avec des enjeux communicationnels multiples}

Quels sont les enjeux communicationnels du rapport de visite? Ils se distinguent en fonction du destinataire : l'institution, le stagiaire et le formateur lui-même.

Pour l'institution, c'est une pièce d'un dossier qui permet l'évaluation finale du stagiaire et, à moyen terme, sa titularisation ou non.

1. Les rubriques sont fixes quel que soit le moment de l'année où la fiche est remplie. 
Pour le stagiaire, c'est un document attestant de la visite et consignant les éléments pour une évaluation sommative. Mais c'est aussi la mémoire de la visite, avec un condensé de l'entretien oral avec le formateur, ainsi qu'un document qui devrait être utile à la préparation de ses stages ultérieurs, pour améliorer sa pratique.

Pour le formateur, cet écrit revêt une double fonction, formative (quand il adresse des recommandations au stagiaire) et évaluative (dans sa dimension institutionnelle); il est aussi une mémoire de la visite pour en rendre compte lors de réunions-bilans des formateurs du groupe de formation après chaque stage.

À la croisée de ces cadres communicationnels, le formateur est reconnu comme l'expert : sur quels savoirs s'appuie-t-il pour rendre compte de la pratique des stagiaires?

\section{Constitution du corpus}

Nous avons constitué notre corpus en sélectionnant les variables suivantes :

- le choix du stage : il s'agit du stage dit filé ${ }^{2}$;

- le moment de la visite : nous avons sélectionné des rapports effectués lors des premières visites (fin octobre-début novembre), qui sont habituellement plus des visites conseil que des visites avec une dimension d'évaluation sommative;

- le niveau : nous avons choisi des rapports de visite en cycle 3 (CE2, CM1 et CM2 de l'école primaire française);

- les catégories de formateurs : nous avons veillé à ce que différentes catégories de formateurs soient représentées, en sélectionnant 4 rapports d'enseignants maitres-formateurs (EMF), 6 rapports d'enseignants du second degré (PIUFM), 2 rapports d'enseignants-chercheurs (MC).

Nous avons précisé ce corpus en sélectionnant une rubrique : «conduite de séances» qui, pour nous, revêt un statut spécifique et central. En effet, c'est une rubrique qui est le lieu de la description et du commentaire de l'action du stagiaire et donc le lieu privilégié de mise en mots des savoirs convoqués par le formateur.

2. Cette modalité de stage conduit les professeurs des écoles stagiaires (PE2) à prendre en charge une classe un jour de la semaine tout au long de l'année. 
Cette étude consiste en un point de départ exploratoire à propos d'un écrit dont nous avons vu qu'il est particulièrement complexe du point de vue communicationnel; nous avons donc fait le choix d'un corpus assez restreint devant nous permettre un premier examen des contenus de savoirs que des formateurs considèrent assez fondamentaux pour être figés dans un texte écrit. Cette perspective qualitative a pour ambition de poser de premières hypothèses quant à la nature des savoirs que les formateurs sélectionnent pour les professeurs des écoles en formation.

\section{Les savoirs convoqués dans les rapports de visite}

Pour un formateur, commenter les savoirs montrés des formés est un exercice délicat, car il demande de décortiquer un geste professionnel dense, complexe, articulant au moins trois dimensions : la connaissance des contenus à enseigner, la connaissance des situations d'enseignement, la connaissance des postures effectives d'appropriation des apprenants. Notre objectif consiste à repérer quels savoirs les formateurs convoquent pour commenter les savoirs et savoir-faire des professeurs stagiaires. Il nous faut donc construire un outil propre à l'analyse de ces savoirs. La littérature à notre disposition montre une grande diversité de typologies et de définitions, dont certaines insistent sur le caractère global de l'acte d'enseigner et sur l'intérêt de considérer les gestes de l'enseignant comme des gestes didactiques (Aeby-Daghé et Dolz, 2008). Dans ce cadre, l'objet à enseigner devient le centre d'une construction dite «didactique» allant de la mise en place de dispositifs à l'évaluation pour devenir un objet effectivement enseigné.

Pour d'autres chercheurs, l'analyse de la transformation des contenus de savoir en savoirs adaptés au public concerné implique la distinction entre différents types de transposition. C'est la position par exemple de Perrenoud (2004) qui propose la caractérisation suivante :

Les travaux de Verret (1975) et des didacticiens, et tout d'abord ceux de Chevallard (1991) ont contribué à construire et à faire connaitre la notion de transposition didactique. Appliquée aux savoirs, l'idée de base est simple : sous leur forme native, celle qui prévaut dans les ouvrages et les communications scientifiques, les savoirs savants ne sont enseignables «tels quels» qu'à des étudiants très avancés, qui partagent déjà une bonne partie des acquis de la discipline considérée et disposent des moyens intellectuels requis pour assimiler directement de la théorie. De la même façon, on peut dire que les savoirs ne deviennent 
utilisables dans une action professionnelle, quelle qu'elle soit, qu'au prix d'une transposition pragmatique. Les deux concepts sont cousins, mais distincts. On peut considérer la transposition didactique comme une forme de transposition pragmatique, avec une différence cependant : l'apprêt didactique vise en priorité des apprenants et répond aux contraintes du système didactique : le temps des études et son découpage en années, semestres et périodes, le contrat tacite qui se noue entre le maitre et l'élève, le rapport scolaire au savoir, l'évaluation. La transposition didactique porte sur les savoirs à enseigner. La transposition pragmatique telle qu'elle est envisagée ici opère sur les savoirs pour enseigner, ceux qui fondent les décisions et les actions pédagogiques et didactiques. (Perrenoud, 2004, p. 142)

Les rapports de visite étant (plus ou moins) la récapitulation d'une situation d'échanges et, dans tous les cas, une mémoire sous forme de condensé de cette situation vécue, notre analyse des commentaires sur les gestes professionnels s'est fondée sur la présence de certains mots-clés caractérisant les situations d'enseignement/apprentissage observées. Ce premier regard sur le matériau à notre disposition nous a conduit à classer les verbalisations des formateurs en fonction de la distinction de Perrenoud (savoirs à enseigner vs savoirs pour enseigner) en toute conscience du risque interprétatif pris dans notre tentative de découpage du propos écrit des formateurs.

L'observation fine des remarques, contenant par exemple de nombreuses références aux contenus déclinés dans les programmes officiels, a imposé un affinement de notre outil, ce que nous avons fait en prenant en compte la typologie des savoirs proposée par Sabine Vanhulle (2009).

Cette dernière distingue d'abord les savoirs de référence, «scripturalisés », et de ce fait même bénéficiant d'une certaine légitimité. Il s'agit d'une part des savoirs académiques «proposés aux étudiants comme des références scientifiques pour comprendre et concevoir leur profession» (2009, p. 170). Il s'agit d'autre part des savoirs institutionnels (issus des savoirs académiques et formalisés par exemple dans les programmes, déterminant les attentes de l'école et de la société à une époque historique donnée). Savoirs académiques comme savoirs institutionnels peuvent concerner autant des savoirs à enseigner que des savoirs pour enseigner. Les savoirs académiques sont, par exemple, les savoirs issus de la psycholinguistique en matière de compréhension et aussi les savoirs disponibles en didactique de la lecture. Quant aux savoirs institutionnels, ils sont en lien avec les savoirs académiques 
et peuvent également concerner tant les savoirs à enseigner que pour enseigner. Toutefois, dans les programmes français de 2008, pour la première fois, le choix des méthodes et des démarches est laissé aux enseignants ( «lls [les programmes nationaux de l'école primaire] laissent cependant libre le choix des méthodes et des démarches, témoignant ainsi de la confiance accordée aux maitres pour une mise en œuvre adaptée aux élèves », peut-on lire dans le préambule du Bulletin officiel hors série $n^{\circ} 3$ du 19 juin 2008) et aucun savoir institutionnel pour enseigner n'apparait explicitement ${ }^{3}$.

Ce sont ces savoirs de référence (académiques et institutionnels) dont il sera essentiellement question dans les lignes qui suivent.

Vanhulle ajoute à cette catégorie des savoirs de référence les savoirs pratiques, ceux tirés par les stagiaires de l'observation des pratiques enseignantes, et des savoirs expérientiels plus implicites et intimement liés au vécu de chacun. Cette catégorie des savoirs pratiques se retrouve dans le corpus étudié par Vanhulle, qui est constitué d'écrits réflexifs d'enseignants stagiaires. En ce qui nous concerne, nous avons recherché des traces verbales de savoirs pratiques des formateurs : font-ils explicitement référence à leur propre expérience d'enseignement dans leur rôle de «conseiller pédagogique» lors de la rédaction du rapport?

\section{Les savoirs à enseigner}

Ils apparaissent massivement en tant que savoirs institutionnels, et très peu en tant que savoirs académiques. Dans une fiche de visite, ils ne sont pas détaillés mais sont présentés globalement : «Les séances sont conduites dans le respect des programmes scolaires, de la situation école et de la situation classe» (MC1). Mais dans les autres fiches ils sont énumérés, selon les cas, en fonction de trois entrées qui peuvent s'articuler : les disciplines et/ou contenus («poésie»; «géométrie»; «séance de sciences. Le cycle de l'eau»); les objectifs («L'objectif porte sur les tracés des droites parallèles» [EMF1]); les activités («Le maitre dessine un rectangle au tableau et demande aux élèves de carac-

3. Le texte officiel précise cependant le cadre de la liberté annoncée : «La liberté pédagogique induit une responsabilité : son exercice suppose des capacités de réflexion sur les pratiques et leurs effets. Elle implique aussi, pour les maitres, l'obligation de s'assurer et de rendre compte régulièrement des acquis des élèves.» (Préambule du Bulletin officiel hors série $\mathrm{n}^{\circ} 3 \mathrm{du}$ 19 juin 2008.) 
tériser cette figure» [EMF2]). Ils sont très peu expansés. Nous n'avons relevé que deux mentions explicites d'enjeux didactiques : un formateur précise que l'objet de savoir observé se situe dans le cadre élargi d'une séquence didactique («Une séance de musique clôt la demi-journée : les élèves ont travaillé dans les séances précédentes sur le texte de chanson «Quatre saisons» de Grand Corps malade. La définition d'un style musical est abordée » [EMF1]). Un autre formateur évoque une donnée de didactique de la conjugaison pour apporter son point de vue («On pouvait leur faire rappeler la différence entre temps simples et temps composés de l'indicatif» [EMF4]).

Ainsi, les savoirs à enseigner sont recensés en rapport avec la façon dont ils apparaissent dans les programmes, mais sont très peu commentés du point de vue didactique. Les rapports analysés ne contiennent quasiment pas d'éléments qui pourraient aider le stagiaire, à la relecture, à faire des liens avec des éléments vus en cours. Le niveau de transposition choisi pour évoquer les savoirs à enseigner est celui des programmes pour l'école. Nous ne relevons que deux mentions d'objets de savoir dont la formulation peut difficilement être celle de l'école, mais relève plus de celle de savoirs disciplinaires transposés pour la formation («Attention, on est dans une approche grammaticale de la conjugaison, l'objectif est différent : faire les accords $\mathrm{S} / \mathrm{V}$ en remplaçant le GNS par un pronom» [EMF3]; «La maitresse note au tableau le mot isolé sans le référer à son contexte textuel» [PIUMF 3]).

\section{Les savoirs pour enseigner}

Si pour les savoirs à enseigner, les formateurs parlent au nom de l'institution, ce n'est pas le cas pour les savoirs pour enseigner. Nous avons souligné plus haut la particularité des programmes de 2008, qui ne privilégient explicitement aucune position théorique pédagogique ou didactique. Or, dans leur rapport de visite, la majorité des formateurs se réfère à des pratiques pédagogiques que l'on pourrait qualifier de «méthodes actives», sans se situer explicitement ni du côté des savoirs institutionnels, ni du côté des savoirs académiques, puisqu'ils ne renvoient pas à des savoirs de référence précis; leur discours relève d'une position en relation avec une conception pédagogique qui considère l'activité de l'élève comme le point nodal de l'appropriation d'un savoir.

Même si les formateurs concernés ne citent aucun courant de la pensée pédagogique, des références théoriques transparaissent dans leurs écrits. En effet, comme nous allons l'illustrer ci-après, certains 
termes récurrents construisent une constellation dont le noyau semble être les méthodes pédagogiques actives.

Nous retrouvons en premier lieu l'importance de l'activité de l'élève et de l'appui sur la parole de ce dernier :

Il faut que les élèves soient actifs au maximum. (EMF 2a)

À partir de cette séance, il rappelle aux élèves toutes les terminaisons des temps simples : n'est-ce pas aux élèves de le faire? (EMF 4a)

Pensez toujours à susciter des situations-problèmes : on observe les mots en grand nombre, on essaie de les trier puis d'expliquer le classement mis en place, on multiplie alors les exemples pour arriver à une règle de construction des mots dérivés. Il faut rendre les élèves actifs! (EMF 2b)

Non seulement l'élève doit être actif, mais son activité doit avoir du sens :

La situation proposée est authentique, le travail bien contextualisé et porteur de sens. (PIUFM 6)

Pensez à rebondir sur les interventions des élèves lorsqu'elles sont porteuses de sens pour atteindre l'objectif visé. (EMF 1)

La question du fonctionnement des groupes est aussi évoquée :

Certains principes du travail de groupes ne sont pas respectés par le maitre puisqu'il effectue la mise en commun sans faire appel aux rapporteurs de groupes mais avec tout le groupe-classe. (EMF 4b)

De retour en classe, par groupes de travail ils mettent en herbier les échantillons ramenés en vue de les identifier par la suite. Ils se révèlent passionnés par les activités proposées et, curieux, commencent immédiatement le travail d'identification. (PIUFM 2)

Le rôle de la trace écrite et son élaboration sont également l'objet de plusieurs remarques :

La géométrie doit obligatoirement passer par l'observation, la manipulation et le tracé des élèves! Pour arriver à la définition, il faut observer toutes sortes de droites, et par un tri trouver celles qui ne sont pas parallèles et celles qui le sont. On peut alors expliquer pourquoi et on trouve la définition des droites parallèles. La trace écrite est ainsi toute faite. (EMF2)

Les élèves sont actifs et sollicités à bon escient; la trace écrite est élaborée en commun. (PIUFM 6) 
Dans des modalités d'actualisation variées (la mention explicite [EMF 2a], le commentaire [EMF 4], l'exemple [PIUFM 6], le compte rendu [PIUFM 2], le script d'actions à visée de généralisation [EMF 2b]), les formateurs concernés s'appuient sur des théories de l'apprentissage centrées sur le sujet.

Du point de vue de l'activité, on constate le lien effectué entre le sens de celle-ci et les situations proposées. Ainsi, il semble que des concepts comme ceux de mobilisation (Léontiev, 1984; Charlot, Rochex et Bautier, 1992), de situations authentiques (Aeby-Daghé et Dolz, 2008), ou de situation-problème soient convoqués.

La notion d'activité telle que définie par Léontiev (1984) comporte trois dimensions qui sont présentes dans les commentaires des enseignants :

- le mobile, qui incite le sujet à agir;

- l'action, définie par son but, par un résultat à atteindre ;

- et l'opération déterminée comme la mise en œuvre des moyens afin d'atteindre le but visé.

Ces dimensions sont concrétisées par le compte rendu de PIUMF 2 par exemple :

La séance débute par une confrontation des conclusions des travaux de recherche concernant l'étude des arbres de la cour de récréation. Les résultats sont affichés au tableau sous forme d'herbiers de façon à amener les élèves à prendre conscience des divergences concernant certaines espèces et à expliciter l'ensemble des critères à prendre en compte pour identifier une espèce inconnue [...]. Le maitre construit avec eux une démarche pour identifier une espèce inconnue, qu'ils devront mettre en place lors d'une sortie à un verger près de l'école; ils auront à reconnaitre trois espèces (pommier, noyer, châtaignier). Par ailleurs, une fiche de recherche à compléter leur est distribuée.

Pour cette sortie les élèves sont répartis en trois ateliers; un parent d'élève est mis à contribution pour l'encadrement. Il s'avère cependant que les trois espèces censées être inconnues ne le sont pas du tout pour eux. Aussi, de façon à éviter une perte d'intérêt, nous invitons le maitre à proposer aux élèves de se reporter sur les espèces inconnues de la haie qui borde le verger...

De retour en classe, par groupes de travail, ils mettent en herbier les échantillons ramenés en vue de les identifier par la suite. Ils se révèlent passionnés par les activités proposées et, curieux, commencent immédiatement le travail d'identification. 
Dans ces lignes, le formateur met effectivement en avant l'intérêt de la finalisation de l'action pour les élèves : ils sont mobilisés parce qu'ils sont impliqués positivement dans le projet («ils se révèlent passionnés»). Il insiste bien sur le fait que l'élève ne s'engage dans un travail cognitivement couteux que s'il y trouve de l'intérêt («de façon à éviter une perte d'intérêt, nous invitons...»). Les objectifs d'apprentissage ciblés semblent donc devoir être atteints parce qu'il y a mobilisation cognitive. Les élèves s'engagent dans l'action pour résoudre la situation problème : «Le maitre construit avec eux une démarche pour identifier une espèce inconnue qu'ils devront mettre en place lors d'une sortie à un verger près de l'école.»

Par ailleurs, un enseignant cité ci-dessus (PIUFM 6) valorise une situation «authentique» comme étant porteuse de sens : nous retrouvons dans ses propos une remarque d'Aeby-Daghé et Dolz qui évoquent «un tissage avec la vraie vie» (2008, p. 138) privilégié par les enseignants qu'ils ont observés. Pour ces enseignants,

une séance réussie est une séance où le savoir en jeu est relié à un thème considéré comme susceptible d'intéresser les élèves [...]. Est valorisée l'authenticité : authenticité de la situation, du document, du témoignage... (Aeby-Daghé et Dolz, 2008, p. 137)

Enfin, dans la citation d'EMF 2b, il est question de résolution de problème, dont J.-F. Halté écrit en 1992 qu' «elle est devenue ces dernières années, dans toutes les didactiques, une référence centrale de l'apprentissage» (p. 82). Renvoyant aux travaux de d'Hainault (1983), Halté rappelle que la résolution de problèmes de haut niveau oblige à inventer du nouveau, et que c'est en cela que les obstacles cognitifs favorisent les apprentissages.

Du point de vue du mode d'apprentissage lui-même, se dégage une conception de la construction du savoir et du sujet du point de vue d'une psychologie sociale (Vygotski, 1985; Bruner, 1998, Perret Clermont, 1974) : l'élève n'apprend pas tout seul, le travail cognitif présente une dimension sociale et l'apprentissage est le fruit de processus sociocognitifs que l'enseignant peut favoriser en construisant des scénarios propres à la structuration cognitive des élèves. La médiation de l'autre semble centrale, qu'il s'agisse de l'enseignant («les élèves sont sollicités à bon escient» [PIUFM 6]) ou des pairs. À la médiation des pairs sont liées les mentions explicites du travail de groupe. Ce dernier est donné comme une modalité constructive à condition du respect de «principes» (EMF 4b); PIUFM 2 parle de «confrontation», ce qui renvoie 
aux premiers travaux sur l'apprentissage en groupe et à une des thèses fondatrices de l'interactionnisme proposée par G. H. Mead (1963) : le sens est interactif, la construction cognitive est liée aux interactions sociales.

Selon les formateurs dont nous avons analysé les rapports, la place accordée aux savoirs pour enseigner semble délivrer un message fort : le savoir disciplinaire de l'enseignant ne suffit pas à l'acte d'enseigner. C'est sa capacité de médiation, sa capacité à développer des relations entre pairs, mais aussi sa capacité à considérer le langage comme le moyen de renforcer, conforter le concept, de passer de l'exemple à la généralisation et de verbaliser sa pensée (par exemple autour des échanges et des modalités d'élaboration des traces écrites) qui fondent sa compétence.

\section{Conclusion}

Ce travail repose sur l'étude d'un corpus relativement restreint et, de plus, dissocié de la situation orale d'entretien avec laquelle il est en rapport. Nous avons conscience de ces limites méthodologiques et de la nécessité d'études de plus grande ampleur, sur un corpus quantitativement beaucoup plus important et avec une prise en compte de l'articulation entre l'oral et l'écrit liée à la visite au stagiaire.

Cependant, l'étude ci-dessus a permis un début d'éclairage sur les conceptions du savoir, de sa transmission, de sa construction dans ce discours professionnel qu'est le rapport de visite.

Premièrement, nous avons constaté un déséquilibre entre des observations ou des conseils à propos de la «médiation didactique» (qui proposeraient par exemple des pistes pour un découpage et une hiérarchisation d'un contenu disciplinaire afin d'en construire des objets évolutifs de connaissance pour les élèves) - et les propos concernant la «médiation pédagogique» (qui commentent la place du groupe, la place de l'enseignant, le rôle du langage dans l'appropriation des savoirs par l'élève). Le premier axe est quasiment absent, alors que le second est très présent.

Deuxièmement, dans la plupart des cas, les critères d'évaluation pris par les formateurs-scripteurs ne se réfèrent pas explicitement à des théories pédagogiques, mais nécessitent une activité d'inférence de la part du lecteur. Les rapports peuvent être ainsi lus de deux manières. Le stagiaire peut faire le lien entre les jugements du formateur et les théories afférentes, mais il peut aussi prendre les remarques du 
formateur comme les traces d'une doxa à laquelle il aura tout intérêt à se conformer pour voir valider sa formation.

Troisièmement, un peu plus haut, nous nous sommes posé la question de la présence des savoirs pratiques des formateurs dans les rapports de visite et des références à leur propre expérience d'enseignement. Nous pouvons constater que ces derniers ne font pas mention de leur expérience personnelle (mais peut-être le font-ils à l'oral en tant qu'enseignants ou anciens enseignants dans le premier ou le second degré). Cette absence de référence à l'expérience personnelle est sans doute l'indice de formateurs ayant théorisé une pratique, pratique ellemême en appui sur les théories de l'apprentissage qu'ils médiatisent de façon concrète et contextualisée dans leurs remarques écrites.

\section{RÉFÉRENCES BIBLIOGRAPHIQUES}

AdAm J.-M. (2005) : La linguistique textuelle, Introduction à l'analyse textuelle des discours, Paris, Armand Colin.

Aeby-Daghé S. et Dolz J. (2008) : «Des gestes didactiques fondateurs aux gestes spécifiques à l'enseignement-apprentissage du texte d'opinion» dans D. Bucheton et O. Dezutter (dir.), Le développement des gestes professionnels dans l'enseignement du français, Bruxelles, De Boeck \& Larcier, p. 83-105.

BRUNER J. (1998) : Le développement de l'enfant : savoir faire, savoir dire, Paris, PUF.

Charlot B., Rochex J.-Y. et Bautier E. (1992) : École et savoir dans les banlieues...et ailleurs, Paris, Armand Colin.

D'Hainaut L. (1983) : Des fins aux objectifs, Paris, Nathan.

Halté J.-F. (1992) : La didactique du français, Paris, PUF.

Leontiev A. (1984) : Activité, conscience, personnalité, Moscou, Éditions du Progrès.

Mead G. H. (1963) : L'esprit, le soi, la société, Paris, PUF.

Perrenoud P. (2004) : «Les sciences de l'éducation proposent-elles des savoirs mobilisables dans l'action?», dans C. Lessard, M. Altet, L. Paquay et P. Perrenoud (dir.), Entre sens commun et sciences humaines : quels savoirs pour enseigner?, Bruxelles, De Boeck, p. 139-158.

Perret Clermont A.-N. (1974) : La construction de l'intelligence dans l'interaction sociale, Genève, Peter Lang.

VANHUlLE S. (2009) : «Savoirs professionnels et construction sociodiscursive de l'agir», dans L. Filliettaz (dir.), Bulletin Vals-Asla, n ${ }^{\circ} 90$, p. 167-188.

Vygotski L. (1985) : Pensée et langage, Paris, Messidor/Éditions sociales. 